\title{
Acolhimento Familiar em Portugal e Espanha: Uma Investigação Comparada sobre a Satisfação dos Acolhedores
}

\author{
Family Foster Care in Portugal and Spain: A Comparative Research \\ on the Satisfaction of Foster Carers
}

\author{
Paulo Delgado*, a, Mónica López ${ }^{b}$, João Carvalho ${ }^{c}$ \& Jorge F. Del Valle ${ }^{d}$ \\ ${ }^{a}$ Instituto Politécnico do Porto, Porto, Portugal, ${ }^{b}$ University of Groningen, Groningen, Holanda, \\ ${ }^{c}$ Instituto Universitário da Maia, Porto, Portugal \& ${ }^{d}$ Universidad de Oviedo, Oviedo, España
}

\begin{abstract}
Resumo
Este estudo apresenta uma comparação entre as experiências de famílias de acolhimento de Portugal e Espanha, avaliando-se a sua satisfação com a informação recebida antes do acolhimento; a preparação da criança para o acolhimento; os apoios técnico e financeiro; e a evolução do processo de acolhimento. Pretende-se contribuir para melhorar e promover a medida de acolhimento familiar nos dois países. Os processos de acolhimento familiar dependem de fatores históricos e culturais que produzem diferenças importantes nos diversos contextos internacionais. Assim, foram realizadas entrevistas em duas amostras aleatórias constituídas por 52 famílias de acolhimento em Portugal e 46 na Espanha, cujos resultados foram comparados através de testes estatísticos paramétricos e não paramétricos. Os acolhedores, em ambos os países, mostraram um elevado grau de satisfação com o processo de acolhimento. No entanto, em Portugal, existe maior preocupação com o fraco apoio financeiro, as informações não fornecidas pelos serviços de acolhimento ou a má preparação da criança para o acolhimento. Por fim, são apresentadas sugestões para a melhoria da gestão e implementação desta resposta social.

Palavras-chave: Bem-estar da criança, acolhimento familiar, satisfação dos acolhedores, estudo comparativo.
\end{abstract}

\begin{abstract}
This study provides a comparison between experiences of Portuguese and Spanish foster families and evaluates their satisfaction with the information received before the host; the preparation of the child for the foster care; the technical and financial support; and the evolution of the foster care process. It aims to contribute to improving implementation and development of this social response in both countries. The foster care processes depend on historical and cultural factors that have significant differences in various international contexts. Thus, two random samples of 52 foster families in Portugal and 46 in Spain were interviewed, and the results were compared using parametric and non parametric statistical tests. The carers, in both countries, show a high degree of satisfaction with the foster care process. However, in Portugal there is greater concern about the poor financial support, information not provided by the foster care services or poor preparation of the child for childcare. Finally, the study presents suggestions for improving the management and implementation of this social response.

Keywords: Child well-being, foster care, carers' satisfaction, comparative research.
\end{abstract}

Uma das atividades mais descuidadas no campo da proteção da infância é a análise do funcionamento dos programas de acolhimento na perspetiva dos próprios utilizadores (Andersson, 2001; Curran \& Pecora, 1999). A principal razão para esta lacuna poderá ser a dificuldade

\footnotetext{
*Endereço para correspondência: Instituto Politécnico do Porto, Escola Superior de Educação, Unidade Técnico-Científica de Ciências da Educação, Rua Dr. Roberto Frias, 602, Porto, Portugal 4200-465.E-mail: pdelgado@ ese.ipp.pt, m.lopez.lopez@rug.nl, jcarvalho@ismai.pt e jvalle@uniovi.es
}

metodológica com que os investigadores têm de lidar no momento de recolher o feedback dos envolvidos nesta medida (Baker, 2007; Berrick, Frasch, \& Fox, 2000).

Apesar das possíveis dificuldades que coloca, nomeadamente o acesso consentido às famílias, escutar os acolhedores é uma atividade essencial para o desenvolvimento e aperfeiçoamento dos serviços de acolhimento familiar, para o desenvolvimento teórico sobre a intervenção e, inclusivamente, para o empowerment dos envolvidos (Baker, 2007; Kirton, Beecham, \& Ogilvie, 2007). Mais, avaliar regularmente a satisfação dos acolhedores pode facilitar a identificação precoce de diversos problemas que 
Delgado, P., López, M., Carvalho, J. \& Del Valle, J. F. (2015). Acolhimento Familiar em Portugal e Espanha: Uma Investigação Comparada sobre a Satisfação dos Acolhedores.

podem colocar em risco o acolhimento, possibilitando a intervenção no início dos mesmos e melhorar, deste modo, o seu prognóstico. Esta prática pode ajudar a prevenir a rutura do acolhimento familiar e as consequências negativas para as crianças ou jovens e famílias (López, Del Valle, Montserrat, \& Bravo, 2011), contribuindo ainda para reduzir o custo dos programas (Whenan, Oxlad, \& Lushington, 2009).

Parece evidente que o estudo da experiência das famílias de acolhimento merece urgentemente a atenção de investigadores dedicados ao sistema de proteção infantil, estudo ainda mais necessário em países como Portugal ou Espanha, onde a implementação destes programas continua a aguardar o impulso necessário, de modo a promover o seu desenvolvimento para níveis idênticos aos de outros países ocidentais (Del Valle, López, Montserrat, \& Bravo, 2009; Delgado, 2013). A ideia de medir a satisfação das familias de acolhimento é, por um lado, um exercício que contribui para que as escassas famílias, que fazem parte do programa, possam desenvolver nexos de vinculação com a sua atividade. Por outro lado, pode também ser útil para a planificação do recrutamento das futuras famílias acolhedoras já que, por exemplo, conhecer as necessidades das famílias acolhedoras atuais pode ajudar a desenhar campanhas mais atrativas e intervenções de apoio mais eficazes, o que posteriormente se traduzirá num recrutamento mais bem- sucedido e na permanência e retenção dessas famílias.

Apesar do crescente número de investigações comparadas sobre os sistemas de proteção infantil, a maioria dos estudos existentes sobre o acolhimento familiar ocorreram nos países anglo-saxônicos, fundamentalmente no Reino Unido, nos Estados Unidos e na Austrália (e.g. Begg, 2011; Courtney \& Iwaniec, 2009; Esping-Andersen et al., 2012; Gilbert, Parton, \& Skivenes, 2011; Healy, Lundström, \& Sallnäs, 2011). Todavia, os países ibero-americanos possuem certas particularidades culturais, históricas e políticas, que tornam questionável a replicabilidade dos resultados daqueles estudos para estas realidades. Neste sentido, cremos que a investigação cooperativa internacional, numa lógica comparada, constitui uma ferramenta de grande utilidade.

Tendo em conta os argumentos expostos, comparamos as experiências de famílias de acolhimento de Portugal e Espanha, avaliando-se a sua satisfação com o acompanhamento e apoio prestado pela Equipa de Acolhimento (constituída pelos técnicos das entidades administrativas de enquadramento e seguimento de cada caso); com a informação recebida antes do acolhimento; com a preparação da criança para o acolhimento; com os apoios técnico e financeiro; e com os resultados absolutos e relativos do processo de acolhimento, pretendendo-se contribuir para melhorar e promover a medida de acolhimento familiar nos dois países.

Embora os dois espaços territoriais representem o contexto de estudo, também serão tratados como unidade de análise, no momento de examinar como a aplicação desta medida pode ser afetada por certas características do país em questão.

A seguir apresenta-se uma descrição geral dos dois sistemas de proteção necessária para a posterior interpretação dos resultados.

\section{O Acolhimento Familiar nos Sistemas de Proteção dos Países Ibéricos}

De acordo com a popular classificação de regimes de estado social, definida por Esping-Andersen (1993, 2000), Portugal e Espanha integram o denominado modelo familista, em parceria com a Itália e a Grécia. Este modelo caracteriza-se pela importância da família na solidariedade social, a quem cabe substituir a mediação deficitária dos Estados (Santos \& Ferreira, 2001). A adesão tardia aos sistemas de segurança social resulta da evolução política e histórica nestes países, com processos de transição de regimes políticos autoritários para regimes políticos democráticos, ocorrida apenas na década de 70 do século passado (Leibfried, 1993).

As políticas de proteção infantil e as suas práticas refletem de modo evidente as peculiaridades referidas. Ao longo de quase todo o século XX, as grandes instituições de acolhimento, de cariz confessional, são praticamente a única resposta de proteção à infância desprotegida. $\mathrm{O}$ acolhimento familiar, que conta com várias décadas de implantação nos países da Europa central e do norte, apresenta taxas de aplicação que indicam uma lenta e dificil progressão, particularmente no caso portugués (Delgado, 2012).

Em Espanha, o acolhimento familiar sem laços de parentesco, apesar de só ter sido introduzido legalmente no ano de 1987, representa, na atualidade, aproximadamente $20 \%$ do total do acolhimento familiar (Del Valle, Bravo, \& López, 2009). A investigação espanhola demonstra que esta intervenção proporciona uma grande estabilidade e permanência às crianças acolhidas, mantendo-se uma grande parte delas a viver com os acolhedores depois de cumprir os 18 anos ou mesmo sendo adotados (ao todo cerca de $40 \%$ do total; Del Valle, López, et al., 2009); que é reduzido o número de ruturas do acolhimento, especialmente quando a criança se encontra na sua família alargada (López et al., 2011); que existe o prolongamento das estadias, com a consequente disminuição de possibilidades de retorno à família biológica (López, Del Valle, Montserrat, \& Bravo, 2013); e que se prolonga da convivência com os acolhedores uma vez alcançada a maioridade (López et al., 2013). Como é lógico, esta permanência tem a sua contrapartida, representada por um serviço estagnado, com dificuldades de evolução, em que é muito difícil que uma família possa cumprir ciclos consecutivos de acolhimento. Trata-se de um modelo quase adotivo que põe em perigo a sustentabilidade do sistema de acolhimento a médio/longo prazo (Delgado, Carvalho, \& Pinto, 2014). 
Em Portugal, o acolhimento familiar foi formalizado legalmente em 1979. Como consequência de um novo regime de execução do acolhimento familiar (Decreto-Lei $n^{0} 11,2008$ ), operou-se uma classificação restrita do âmbito da medida, permitindo a colocação apenas na família sem laços de parentesco, o que fez diminuir, de modo expressivo, o papel do acolhimento familiar no âmbito das medidas de colocação. Por outro lado, apesar do novo diploma consagrar o alargamento e aprofundamento dos requisitos na seleção dos acolhedores, e apostar no aumento do nível socioeconómico das famílias de acolhimento, não se procedeu à divulgação da medida nem se desenvolveram processos de seleção de novos acolhedores. No que diz respeito à relação entre a idade das crianças e jovens e o tipo de recurso, é surpreendente confirmar que a maioria das crianças entre 0 e 3 anos são admitidos em instituições, restando um número residual que é encaminhado para acolhimento familiar (Instituto da Segurança Social, 2013). Também em Portugal o acolhimento familiar se carateriza pela estabilidade e permanência. São raros os casos de mudança de família de acolhimento, e a criança ou jovem tende a ficar muitos anos acolhida, em muitos casos até aos 18 anos ou alcançar a sua independência, o que sucede com frequência mais tarde. De facto, $63 \%$ das crianças e jovens em acolhimento familiar encontravam-se acolhidos há mais de 4 anos. Se somarmos a esta percentagem aos que se encontravam acolhidos há pelo menos 2 anos, concluímos que $75,4 \%$ das crianças e jovens em acolhimento familiar se encontravam integrados de forma prolongada (Instituto da Segurança Social, 2013).

Alguns traços aproximam os dois países para além da tradição institucionalizadora e da consequente debilidade da divulgação e utilização do acolhimento familiar. Neles se incluem a permanência no acolhimento já referida, a inexistência de campanhas regulares de recrutamento e de seleção de novos acolhedores, a escassez de famílias de acolhimento disponíveis para acolher, o funcionamento de serviços de acolhimento com poucos recursos humanos e materiais, o que dificulta o acompanhamento das colocações e dos acolhedores, e a falta de sistemas estatísticos e de monitorização destas intervenções.

Entre as diferenças mais significativas destaca-se a maior expressão da colocação em acolhimento familiar no caso espanhol e o predomínio do acolhimento em instituição em Portugal; a importância da família alargada e com a maior idade no início do acolhimento das crianças e jovens em Espanha; o perfil dos acolhedores, com idades mais avançadas, um baixo nível educativo e levando a cabo acolhimentos múltiplos, em Portugal (López, Delgado, Carvalho, \& Del Valle, 2014).

\section{Metodologia}

A metodologia seguida neste estudo apresenta uma estratégia qualitativa e quantitativa, com um desenho transversal e utilizando um método de coleta de dados baseado em entrevistas semiestruturadas. Neste artigo são só apresentados e discutidos os resultados obtidos com as questões que pediam uma resposta em escalas ordinais, as quais permitem uma análise estatística quantitativa.

\section{Amostras}

As amostras foram escolhidas aleatoriamente entre as famílias de acolhimento de Portugal (52 famílias correspondendo a cerca de $17 \%$ do total em 2011) e do País Basco, Espanha (46 famílias, em 2009). A idade média dos acolhedores espanhóis era de 47,24 anos $(D P=6,8)$ e das acolhedoras de 46 anos $(D P=5,9)$. A idade média dos acolhedores portugueses era de 56,29 anos $(D P=8,6)$ e das acolhedoras de 54,12 anos $(D P=8,9)$.

Em ambos os países excluíram-se os casos de acolhimento de facto (situações não reguladas pelas entidades administrativas de enquadramento) e pré-adotivos, por se considerarem realidades muito específicas que se afastavam do âmbito do estudo.

\section{Instrumentos, Procedimentos e Variáveis}

Os dados deste estudo foram coletados através de entrevistas semiestruturadas, presenciais ou, nos casos mais distantes, por via telefónica, com um guião elaborado originalmente para a avaliação do acolhimento familiar em Espanha (Del Valle, López, et al., 2009; López, Del Valle, Montserrat, \& Bravo, 2010). Em Espanha, as entrevistas foram realizadas por duas investigadoras do Grupo de Investigação em Família e Infância, tendo as famílias recebido o guião antes da realização da mesma. Em Portugal, o guião da entrevista foi traduzido e adaptado pelos investigadores da Escola Superior do Instituto Politécnico do Porto (Delgado, 2013), os quais realizaram as entrevistas com os acolhedores. Estas tiveram uma duração média de 90 minutos, tendo decorrido em 2009 em Espanha e em 2011 em Portugal.

Em ambos os países levou-se a cabo um pré-teste do guião da entrevista com três acolhedores, que permitiu ajustar a linguagem a utilizar durante a mesma.

Através das entrevistas exploraram-se diversas dimensões da satisfação do(a) acolhedor(a) em relação: (a) à informação recebida antes do acolhimento sobre o processo de acolhimento: deveres, direitos, responsabilidades, sobre os antecedentes de saúde da criança, sobre a história familiar da criança; (b) à preparação da criança para ser acolhida; (c) ao apoio técnico: disponibilidade dos profissionais para responder quando se lhes pede ajuda ou informação; rapidez de resposta dos técnicos quando se solicita informação ou apoio; grau em que os técnicos têm em conta as opiniões e critérios dos acolhedores; frequência dos contactos com os acolhedores; na obtenção de ajuda ou conselho para problemas de comportamento infantil; (d) ao apoio económico: suficiência do apoio económico; facilidade de gestão e acesso às ajudas económicas; pontualidade no pagamento; (e) ao processo de adaptação da criança ou jovem; (f) e aos resultados do acolhimento. $\mathrm{O}$ guião da entrevista foi aprovado pelas entidades administrativas de enquadramento dos dois países, tendo sido 
Delgado, P., López, M., Carvalho, J. \& Del Valle, J. F. (2015). Acolhimento Familiar em Portugal e Espanha: Uma Investigação Comparada sobre a Satisfação dos Acolhedores.

verificados todos os aspetos éticos que decorrem deste tipo de procedimentos, tendo-se obtido o consentimento das famílias de acolhimento para a realização das entrevistas.

As entrevistas abordaram a perceção dos acolhedores sobre os resultados da medida de acolhimento, de dois modos diferentes: em primeiro lugar, pediu-se uma valorização dos resultados da medida de acolhimento familiar em termos absolutos, avaliando o grau de sucesso que os acolhedores estavam tendo com a sua intervenção, numa escala com quatro opções (êxito, alguns resultados, alguns problemas, graves problemas); e em segundo lugar, investigou-se a perspetiva dos acolhedores sobre o que poderíamos chamar de resultados relativos ou do processo do acolhimento familiar, pedindo-lhes que nos dessem a sua opinião, numa escala ordinal (muito má; má, regular, boa, muito boa), sobre as seguintes situações, tanto no início do acolhimento como na atualidade: a relação da criança com os filhos dos acolhedores; a relação da criança com o acolhedor; a relação da criança com a acolhedora; a relação entre a criança e os seus pais; a disposição da criança sobre as visitas da sua família; as consequências das visitas para a criança; as consequências das visitas para os acolhedores; a saúde física da criança; o bem-estar psicológico da criança; as relações sociais da criança; o comportamento da criança; a motivação para o estudo;

Tabela 1

Informação Recebida pelos Acolhedores

Informacão Recebida pelos Acolhedores

Sobre o processo

de acolhimento o rendimento académico; e o comportamento da criança na escola.

\section{Análise Estatística}

Foram utilizadas estatísticas descritivas (média, desvio padrão, etc.) e inferenciais. Estas permitiram a avaliação das diferenças entre os dois países através de testes estatísticos, como o qui-quadrado para analisar a possível (in) dependência entre variáveis nominais; o teste Wilcoxon para amostras relacionadas, para comparar dados de variáveis qualitativas na mesma amostra entre momentos temporais diferentes; o teste Kolmogorov-Smirnov para duas amostras para comparar distribuições de variáveis ordinais; o teste $t$ para a igualdade de médias em variáveis quantitativas; e o teste de Levene para o estudo da homogeneidade das variâncias entre as populações. Para todos os testes considerou-se que o valor máximo do erro tipo I não deveria ultrapassar os 5\%. A análise estatística foi desenvolvida com recurso ao programa SPSS 20.0.

\section{Resultados}

\section{Satisfação com a Informação Recebida Antes do Acolhimento}

Nos três aspetos analisados sobre a informação recebida pelas famílias antes do acolhimento (Tabela 1), verifica-

\begin{tabular}{|c|c|c|c|c|c|c|c|c|c|}
\hline & Espanha & Portugal & Total & Espanha & Portugal & Total & Espanha & Portugal & Total \\
\hline \multirow{2}{*}{ Nula } & 5 & 4 & 9 & 9 & 14 & 23 & 13 & 14 & 27 \\
\hline & $10,9 \%$ & $7,7 \%$ & $9,2 \%$ & $20 \%$ & $27,5 \%$ & $24 \%$ & $28,9 \%$ & $27,5 \%$ & $28,1 \%$ \\
\hline \multirow{2}{*}{ Reduzida } & 3 & 11 & 14 & 15 & 9 & 24 & 14 & 7 & 21 \\
\hline & $6,5 \%$ & $21,2 \%$ & $14,3 \%$ & $33,3 \%$ & $17,6 \%$ & $25 \%$ & $31,1 \%$ & $13,7 \%$ & $21,9 \%$ \\
\hline \multirow{2}{*}{ Suficiente } & 26 & 13 & 39 & 12 & 12 & 24 & 12 & 12 & 24 \\
\hline & $56,5 \%$ & $25 \%$ & $39,8 \%$ & $26,7 \%$ & $23,5 \%$ & $25 \%$ & $26,7 \%$ & $23,5 \%$ & $25 \%$ \\
\hline \multirow{2}{*}{ Boa } & 12 & 24 & 36 & 9 & 16 & 25 & 6 & 18 & 24 \\
\hline & $26,1 \%$ & $46,2 \%$ & $36,7 \%$ & $20 \%$ & $31,4 \%$ & $26 \%$ & $13,3 \%$ & $35,3 \%$ & $25 \%$ \\
\hline \multirow{2}{*}{ Total } & 46 & 52 & 98 & 45 & 51 & 96 & 45 & 51 & 96 \\
\hline & $100 \%$ & $100 \%$ & $100 \%$ & $100 \%$ & $100 \%$ & $100 \%$ & $100 \%$ & $100 \%$ & $100 \%$ \\
\hline $\mathrm{K}-\mathrm{S} z$ & & 0,991 & & & 0,556 & & & 1,774 & \\
\hline$p$ & & 0,201 & & & 0,917 & & & 0,199 & \\
\hline
\end{tabular}


-se que não há diferenças estatisticamente significativas ( $p$ $>0,05$ ) na distribuição das respostas entre os dois países. No entanto, verifica-se que $23,5 \%$ dos acolhedores não receberam informação ou receberam informação reduzida sobre os seus direitos, deveres e responsabilidades, o que constitui um fator potencialmente gerador de tensões, conflitos e até de ruturas das colocações. Esta situação é mais grave em Portugal, com 28,9\%, contra 17,4\% em Espanha. Por contrapartida, em Espanha 82,6\% considera que este tipo de informação é suficiente $(56,5 \%)$ ou boa $(26,1 \%)$, enquanto que em Portugal essa opinião só acontece com $71,2 \%$ dos casos (suficiente: $25 \%$; e boa: $46,2 \%$ ), mas com mais casos de perceções boas em Portugal, porventura demonstrando que estas opiniões dependem mais do trabalho desenvolvido pelas Equipas de Acolhimento do que da organização dos sistemas de informação.

A ausência ou escassez de informação é maior relativamente aos antecedentes físicos e de saúde da criança, uma vez que $49 \%$ dos acolhedores optam pelas duas primeiras opções de resposta (nula ou reduzida). Esta situação revela uma omissão significativa, involuntária ou intencional, que prejudica ou inviabiliza a necessária ponderação das famílias acolhedoras sobre a sua capacidade para acolherem crianças que sejam eventualmente portadoras de problemas de saúde ou com deficiência. Por outro lado, aumenta os riscos de insatisfação com a experiência e de rejeição da criança, uma vez iniciado o acolhimento. É de notar que parece existir uma maior preocupação quanto a estas informações por parte das Equipas de Acolhimento portuguesas $(23,5 \%+31,4 \%=54,9 \%$ em Portugal; $26,7 \%$ $+20 \%=46,7 \%$ ).

A mesma tendência, de falta ou ausência de informação, constata-se no que se refere à história familiar da criança, uma vez que abrange precisamente metade $(50 \%)$ dos acolhedores no conjunto dos dois países. Essa tendência é superior do caso espanhol, onde atinge $60 \%$ das respostas, face aos $42,2 \%$ do caso português. As perceções positivas são também muito mais favoráveis em Portugal, com $35,3 \%$ de boas opiniões contra só 13,3\% em Espanha.

\section{Satisfação com a Preparação da Criança \\ ou Jovem para o Acolhimento}

A preparação da criança para o acolhimento é melhor executada em Espanha (K-S $z=2,971 ; p<0,001)$, segundo as perceções dos acolhedores. No caso português, não houve qualquer preparação em $70,3 \%$ dos casos, o que evidencia processos desarticulados e improvisados, que não podem ser justificados só pela pela urgência da colocação. Em Espanha os números são quase inversos, pois $80 \%$ dos inquiridos declara que a preparação da criança para o acolhimento foi suficiente ou boa.

Tabela 2

Capacidade de Resposta dos Técnicos

\begin{tabular}{|c|c|c|c|c|c|c|c|c|c|}
\hline & \multicolumn{3}{|c|}{$\begin{array}{l}\text { Disponibilidade dos técnicos } \\
\text { para responder quando se } \\
\text { solicita apoio ou informação }\end{array}$} & \multicolumn{3}{|c|}{$\begin{array}{l}\text { Rapidez das respostas dos } \\
\text { técnicos quando se solicita } \\
\text { informação ou apoio }\end{array}$} & \multicolumn{3}{|c|}{$\begin{array}{l}\text { Grau em que os técnicos } \\
\text { tiveram em conta as opiniões } \\
\text { e critérios dos acolhedores }\end{array}$} \\
\hline & Espanha & Portugal & Total & Espanha & Portugal & Total & Espanha & Portugal & Total \\
\hline \multirow{2}{*}{$\begin{array}{l}\text { Nunca ou } \\
\text { raramente }\end{array}$} & 0 & 1 & 1 & 0 & 2 & 2 & 2 & 8 & 10 \\
\hline & $0 \%$ & $1,9 \%$ & $1 \%$ & $0 \%$ & $4 \%$ & $2,1 \%$ & $4,3 \%$ & $15,4 \%$ & $10,2 \%$ \\
\hline \multirow{2}{*}{$\begin{array}{c}\text { Algumas } \\
\text { vezes }\end{array}$} & 3 & 3 & 6 & 2 & 4 & 6 & 5 & 8 & 13 \\
\hline & $6,5 \%$ & $5,8 \%$ & $6,1 \%$ & $4,3 \%$ & $8 \%$ & $6,3 \%$ & $10,9 \%$ & $15,4 \%$ & $13,3 \%$ \\
\hline \multirow{2}{*}{$\begin{array}{l}\text { Com } \\
\text { frequência }\end{array}$} & 18 & 7 & 25 & 19 & 6 & 25 & 13 & 36 & 49 \\
\hline & $39,1 \%$ & $13,5 \%$ & $25,5 \%$ & $41,3 \%$ & $12 \%$ & $26 \%$ & $28,3 \%$ & $69,2 \%$ & $50 \%$ \\
\hline \multirow{2}{*}{ Sempre } & 25 & 41 & 66 & 25 & 38 & 63 & 26 & 0 & 26 \\
\hline & $54,3 \%$ & $78,8 \%$ & $67,3 \%$ & $54,3 \%$ & $76 \%$ & $65,6 \%$ & $56,5 \%$ & $0 \%$ & $26,5 \%$ \\
\hline \multirow{2}{*}{ Total } & 46 & 52 & 98 & 46 & 50 & 96 & 46 & 52 & 98 \\
\hline & $100 \%$ & $100 \%$ & $100 \%$ & $100 \%$ & $100 \%$ & $100 \%$ & $100 \%$ & $100 \%$ & $100 \%$ \\
\hline $\mathrm{K}-\mathrm{S} z$ & & 1,21 & & & 1,06 & & & 2,792 & \\
\hline$p$ & & 0,107 & & & 0,211 & & & 0,001 & \\
\hline
\end{tabular}


Delgado, P., López, M., Carvalho, J. \& Del Valle, J. F. (2015). Acolhimento Familiar em Portugal e Espanha: Uma Investigação Comparada sobre a Satisfação dos Acolhedores.

\section{Satisfação com o Apoio Técnico}

A facilidade de comunicação e de relação com a Equipa de Acolhimento são variáveis que contribuem para um sentimento de confiança, apoio e segurança, permitindo desenvolver uma prática partilhada. Como se observa na Tabela 2, não existem diferenças estatisticamente significativas entre as distribuições de frequências nas variáveis Disponibilidade dos Técnicos e Rapidez das Respostas, em relação aos dois países. Na perspetiva dos acolhedores, as Equipas de Acolhimento manifestam grande disponibilidade para responder em $92,8 \%$ dos casos em que thes solicitam apoio ou informação e as respostas são rápidas, sempre ou com frequência, em 91,6\% das respostas. Contudo, há diferenças no grau em que os técnicos tiveram em conta a opinião dos acolhedores (K-s $z=2,792 ; p<0,001)$. Em
Espanha, esta opinião é tida em conta num grau superior, e é sempre tida em conta em $56,5 \%$ dos casos, ao contrário do que sucede em Portugal, onde a opção "Sempre" não regista qualquer resposta, apesar da opinião ser tida em conta em $69,2 \%$ das respostas.

Há diferença na distribuição da frequência com que os acolhedores são contactados pelos técnicos (K-S $z=1,718$; $p<0,01$ ), a qual é muito maior em Espanha, assim como na frequência da ajuda e aconselhamento sobre problemas de comportamento da criança (K-S $z=1,8 ; p<0,01$ ), que é também superior em Espanha.

\section{Satisfação com o Apoio Económico}

Outro âmbito analisado foi o grau de satisfação dos acolhedores com o apoio económico que recebem pelo acolhimento (Tabela 3).

Tabela 3

Apoios Económicos

\begin{tabular}{|c|c|c|c|c|c|c|c|c|c|}
\hline & \multicolumn{3}{|c|}{$\begin{array}{l}\text { Suficiência do apoio } \\
\text { económico }\end{array}$} & \multicolumn{3}{|c|}{$\begin{array}{l}\text { Facilidade de gestão } \\
\text { e acesso às ajudas }\end{array}$} & \multicolumn{3}{|c|}{$\begin{array}{c}\text { Pontualidade na receção } \\
\text { das ajudas }\end{array}$} \\
\hline & Espanha & Portugal & Total & Espanha & Portugal & Total & Espanha & Portugal & Total \\
\hline \multirow{2}{*}{ Insatisfeito } & 0 & 29 & 29 & 0 & 22 & 22 & 0 & 25 & 25 \\
\hline & $0 \%$ & $56,9 \%$ & $30,2 \%$ & $0 \%$ & $53,7 \%$ & $25,6 \%$ & $0 \%$ & $48,1 \%$ & $25,8 \%$ \\
\hline \multirow{2}{*}{ Satisfeito } & 0 & 22 & 22 & 0 & 18 & 18 & 0 & 25 & 25 \\
\hline & $0 \%$ & $43,1 \%$ & $22,9 \%$ & $0 \%$ & $43,9 \%$ & $20,9 \%$ & $0 \%$ & $48,1 \%$ & $25,8 \%$ \\
\hline \multirow{2}{*}{$\begin{array}{l}\text { Muito satis- } \\
\text { feito }\end{array}$} & 45 & 0 & 45 & 45 & 1 & 46 & 45 & 2 & 47 \\
\hline & $100 \%$ & $0 \%$ & $46,9 \%$ & $100 \%$ & $2,4 \%$ & $53,5 \%$ & $100 \%$ & $3,8 \%$ & $48,5 \%$ \\
\hline \multirow{2}{*}{ Total } & 45 & 51 & 96 & 45 & 41 & 86 & 45 & 52 & 97 \\
\hline & $100 \%$ & $100 \%$ & $100 \%$ & $100 \%$ & $100 \%$ & $100 \%$ & $100 \%$ & $100 \%$ & $100 \%$ \\
\hline $\mathrm{K}-\mathrm{S} z$ & & 1,21 & & & 1,06 & & & 2,792 & \\
\hline$p$ & & 0,107 & & & 0,211 & & & 0,001 & \\
\hline
\end{tabular}

Apuraram-se diferenças na suficiência do apoio económico aos acolhedores (K-S $z=4,889 ; p<0,001$ ), na facilidade de gestão e acesso às ajudas (K-S $z=4,519$; $p<0,001)$, e na pontualidade de receção dessas ajudas (K-S $z=4,723 ; p<0,001$ ), sendo todos estes processos melhor realizados em Espanha. Em Portugal, 56,9\% dos acolhedores estão insatisfeitos com o apoio económico que recebem, ao inverso do que sucede em Espanha, em que a totalidade dos acolhedores está muito satisfeita. Os resultados relativos aos processos de pagamentos e à pontualidade na prestação das ajudas revelam números semelhantes, o que nos permite identificar um expressivo fator de insatisfação no desempenho das funções do acolhimento familiar, no caso português. Pelo contrário, em Espanha, o apoio económico e os processos que lhe estão associados contribuem, de modo claro, para suportar o desempenho dos acolhedores.

\section{Satisfação com o Processo de Adaptação da Criança ou Jovem}

A adaptação da criança ou jovem, comparando várias dimensões da sua vida entre a atualidade e o início do acolhimento, é analisada inferencialmente na Tabela 4. 
Os resultados são semelhantes entre Espanha e Portugal, havendo uma evolução estatisticamente significativa em quase todas as variáveis. As exceções são a relação entre a criança e os filhos dos acolhedores e nas consequências das visitas para os acolhedores, no caso espanhol.
Em Portugal, não há diferenças estatisticamente significativas na relação com os seus pais, na disposição da criança para as visitas com a sua familia de origem, nas consequências das visitas e nas consequências das visitas para os acolhedores.

Tabela 4

Adaptação da Criança

\begin{tabular}{ccc}
\hline Testes Wilcoxon para amostras relacionadas & Espanha & Portugal \\
\hline Relação da criança com os filhos dos acolhedores & $p=0,142$ & $p<0,05$ \\
Relação da criança com o acolhedor & $p<0,05$ & $p<0,01$ \\
Relação da criança com a acolhedora & $p<0,01$ & $p<0,05$ \\
Relação entre a criança e os seus pais & NA & $p=0,082$ \\
Disposição da criança sobre as visitas da sua família & NA & $p=0,971$ \\
Consequências das visistas para a criança & NA & $p=0,982$ \\
Consequências das visitas para os acolhedores & $p=0,619$ & $p=0,194$ \\
Saúde física da criança & $p<0,001$ & $p<0,001$ \\
Bem-estar psicológico da criança & $p<0,01$ & $p<0,001$ \\
Relações sociais da criança & $p<0,01$ & $p<0,001$ \\
Comportamento da criança & $p<0,01$ & $p<0,001$ \\
Motivação para o estudo & $p<0,01$ & $p<0,001$ \\
Rendimento académico & $p<0,01$ & $p<0,001$ \\
Comportamento da criança na escola & $p<0,05$ & $p<0,001$
\end{tabular}

Nota. NA - Não Aplicado.

Verifica-se, pelo exposto, que a perceção dos acolhedores em relação à evolução da criança ou jovem não é positiva em esferas que remetem para a relação com a familia de origem e para as consequências dos contactos, a que acresce, no caso espanhol, a relação da criança ou jovem com os filhos dos acolhedores. Todas as outras dimensões avaliadas revelam uma adaptação positiva, em diferentes áreas da vida da criança ou jovem acolhido, como a integração e relação com a família de acolhimento, as suas relações sociais, a saúde física e o bem estar psicológico, o seu comportamento e o seu percurso escolar.

\section{Satisfação com os Resultados do Acolhimento}

Não há uma diferença estatiscamente significativa (K-S $z=0,698 ; p=0,714)$ na valoração que os acolhedores dão ao cumprimento dos objetivos do acolhimento, entre os dois países. De facto, $68,4 \%$ dos acolhedores de ambos os países declaram que o acolhimento é um êxito, revelando um elevado grau de satisfação com os resultados atingidos. Apenas 2\% reconhecem a existência de graves problemas nos acolhimentos em curso. É também pequeno o grupo de $8,2 \%$ de acolhedores que declara a existência de alguns problemas. Por fim, 21,4\% dos acolhedores assumem a obtenção de alguns resultados. Em suma, estamos face a uma avaliação muito positiva dos resultados obtidos, em ambos os países, não obstante as diferenças que fomos assinalando ao longo da apresentação dos resultados do estudo.

\section{Discussão e Considerações Finais}

A investigação comparativa, aqui apresentada, evidencia a existência de diferenças mas também de muitos pontos comuns na satisfação dos acolhedores com a prestação da sua atividade, entre Espanha e Portugal.

A informação recebida pelos acolhedores sobre a criança acolhida apresenta deficiências que podem dificultar a fase inicial de integração e, inclusive, a continuidade do acolhimento. O padrão é semelhante nos dois países mas acentua-se no caso português relativamente aos antecedentes físicos e de saúde da criança. Os estudos realizados em Portugal revelam que um número significativo de crianças em acolhimento familiar é portadora de deficiência (Delgado, 2013; Departamento de Desenvolvimento Social, 
Delgado, P., López, M., Carvalho, J. \& Del Valle, J. F. (2015). Acolhimento Familiar em Portugal e Espanha: Uma Investigação Comparada sobre a Satisfação dos Acolhedores.

Unidade de Infância e Juventude, 2012; Instituto de Desenvolvimento Social \& Comissão Nacional de Proteção de Crianças e Jovens, 2002).

O número excessivo de casos seguidos por cada técnico, as dificuldades de comunicação e de articulação entre as diversas entidades com responsabilidade na intervenção, bem como a urgência da colocação, podem explicar as falhas detetadas, as quais fragilizam, naturalmente, os pressupostos em que assenta a decisão de propor o acolhimento e a decisão de aceitação por parte dos acolhedores (Daniel, Wassell, \& Gilligan, 1999; Delgado, 2012; Romaine, Turley, \& Tuckey, 2007; Triseliotis, Sellick, \& Short, 1995). Seria necessário analisar em profundidade o quadro institucional e os processos de tomada de decisões nos dois países, para determinar as causas para estas omissões na informação e procurar pautas de melhoria para as fases de preparação e de colocação da criança, no respeito pelos direitos e bem- estar de crianças e acolhedores, de modo a promover a decisão informada e consciente e a satisfação com o acolhimento.

Uma vez iniciado o acolhimento, o estudo revela um acompanhamento com maior contacto, ajuda e aconselhamento no caso espanhol. São diversos os estudos que concluíram que a satisfação com o apoio técnico prestado aos acolhedores aumenta o grau de satisfação com a experiência (Samrai, Beinart, \& Harper, 2011; Sellick, Thoburn, \& Philpot, 2004; Sinclair, Gibbs, \& Wilson, 2004; Triseliotis, Borland, \& Hill, 2000). A investigação prévia pode comprovar que uma das chaves para manter os acolhedores no desempenho deste difícil papel é proporcionar-lhes o apoio necessário (Brown \& Calder, 2000; Hudson \& Levasseur, 2002; Rhodes, Orme, Cox, \& Buehler, 2003). Apurou-se ainda que as interações insatisfatórias e a falta de comunicação com os técnicos da Equipa de Acolhimento podem aumentar o risco de rutura do acolhimento (Rhodes et al., 2003; Triseliotis, Borland, \& Hill, 1998).

Ou seja, para lá da facilidade de comunicação e de relação com a Equipa de Acolhimento, que se comprova existir nos dois países em análise, é necessário que as famílias de acolhimento recebam apoio eficaz para a resolução dos problemas que surjam no decurso da estadia da criança ou jovem. A Equipa de Acolhimento deve reunir-se regularmente com os acolhedores e com a criança acolhida, e não apenas nas situações de crise. O acompanhamento tem que ser contínuo, atento, perspicaz, devendo ser um espaço de diálogo, de partilha e construção conjunta na tomada de decisão, no respeito pelas competências e pelos papéis de cada um, o que exige tempo, saber, recursos e disponibilidade (Fulcher \& McGladdery, 2011).

No acolhimento familiar português a delegação de responsabilidades na família de acolhimento é muito mais acentuada do que no caso espanhol, o que denota fragilidades no acompanhamento do trabalho dos acolhedores, por um lado, e no controlo e supervisão das colocações, por outro. A escassez de recursos humanos e materiais pode ser uma explicação para esta realidade. Uma vez colocada, a criança integra-se na família acolhedora e o seu caso é, com frequência, esquecido. Este esquecimento constitui uma ameaça para o seu bem estar e segurança e negligencia o desempenho e as eventuais dificuldades sentidas pelos acolhedores.

A satisfação com o apoio económico indica uma tendência idêntica, de maior satisfação na região espanhola. Neste domínio, a agilidade dos processos e a pontualidade na prestação dos apoios contribui para a satisfação com o acolhimento, assim como o montante desses pagamentos (Amorós \& Palacios, 2004; Berridge, 1999; Kirton, Beecham, \& Ogilvie, 2004; Maluccio, Ainsworth, \& Thoburn, 2000; Sinclair et al., 2004; Triseliotis et al., 2000; Verity, 1999). O estatuto socioeconómico e o nível de habilitações podem influenciar o modo como os acolhedores encaram os apoios, quanto ao seu valor e quanto aos processos de gestão (Delgado, 2013). Assim, as famílias de acolhimento com mais dificuldades económicas e dependentes dos apoios para poderem prosseguir o acolhimento, poderão revelar mais insatisfação quanto à pontualidade do pagamento e ao montante desses apoios.

No que diz respeito às mudanças verificadas ao longo do processo de acolhimento na adaptação da criança ou jovem, verifica-se que, no caso português, a evolução da criança é positiva em todos os parâmetros, excluindo os associados à relação ou contacto com a família de origem. $\mathrm{O}$ acolhimento familiar demonstra ser um contexto adequado para o desenvolvimento da criança nos domínios principais da sua vida, como o percurso escolar, a saúde a a integração social, mas este desenvolvimento implica o afastamento da família biológica, intensificando-se a integração na família de acolhimento e o tempo de permanência. Um desafio passa, deste modo, pelo trabalho com a família de origem, tendo em vista a efetiva recuperação das suas responsabilidades parentais, nos casos em que seja viável, de modo a incentivar e apoiar a alteração do quadro deficitário que obrigou à retirada da criança. Só com o desenvolvimento de estratégias que incentivem a cooperação das famílias para a mudança, o processo de acolhimento familiar pode ser uma medida temporária, no respeito por limites temporais ajustados ao desenvolvimento do melhor projeto de vida para a criança acolhida.

Os resultados absolutos do acolhimento são muito positivos nos dois países. Em Espanha, a satisfação com os processos de acompanhamento e com o apoio prestado pela Equipa de Acolhimento surge associada ao cumprimento com sucesso dos objetivos do acolhimento, isto é, dos seus resultados. Pelo contrário, no caso português, os resultados são considerados como um êxito em $75 \%$ dos casos, apesar do grau de satisfação com os processos ser menor do que na região espanhola. A razão para esta diferença pode justificar-se pelo perfil dos acolhedores portugueses, que terão expetativas mais baixas em relação ao apoio e à sua participação efetiva no programa, em parceria com a Equipa de Acolhimento. A sua intervenção está centrada na relação com a criança e a sua integração, revelando 
uma postura mais fechada no que diz respeito à relação com o exterior, quer com os serviços sociais, quer com a família de origem. Os resultados obtidos com a criança são gratificantes e geradores de uma enorme satisfação com o acolhimento, apesar do funcionamento irregular e com lapsos do sistema de proteção. As manifestações de afeto, a alegria da criança e o seu desenvolvimento, são referidos como as principais recompensas de um trabalho exigente e difícil.

A escassez de recursos humanos e materiais pode ser uma explicação para a fragilidade do acompanhamento do trabalho dos acolhedores. Uma vez colocada, a criança integra-se na família acolhedora e o seu caso é, com frequência, esquecido. Este esquecimento constitui uma ameaça para o seu bem- estar e segurança, e negligencia o desempenho e as eventuais dificuldades sentidas pelos acolhedores.

No caso espanhol, o elevado nível de satisfação dos acolhedores com o processo de acompanhamento não está associado a resultados tão positivos quanto ao cumprimento dos objetivos do acolhimento, revelando, provavelmente, um perfil de acolhedor mais crítico, mais informado e mais consciente do seu papel enquanto cuidador temporário das crianças que acolhe.

Em suma, podemos concluir que a satisfação dos acolhedores com os processos do acolhimento não gera necessariamente uma satisfação equivalente relativamente aos resultados. Nos dois casos em estudo podemos apurar tendências distintas. Na região espanhola, uma maior satisfação com os processos do que com os resultados, apesar destes últimos serem também positivos. No caso português, uma maior satisfação com os resultados, apesar das críticas aos processos. Nos dois casos, encontramos como padrão semelhante a adaptação positiva da criança, em dimensões essenciais da sua vida, entre a atualidade e o início do acolhimento.

Por fim, para além das limitações normalmente associadas ao método da entrevista e ao proceso de amostragem, importa referir que não apuramos qualquer relação demonstrável neste estudo entre uma menor satisfação com o acompanhamento e apoio prestado pela Equipa de Acolhimento e os casos identificados com graves problemas ou alguns problemas. De modo idêntico, não foi possível determinar se os resultados associados ao êxito surgem sempre associados à satisfação com o acompanhamento e o apoio prestado pela Equipa de Acolhimento. Para investigação futura, seria interessante averiguar qual a relação que se pode estabelecer entre a satisfação com os processos e com os resultados com o tipo de adaptação da criança entre o início do acolhimento e a atualidade. Dito de outro modo, investigar por exemplo até que ponto uma evolução negativa da criança aumenta a insatisfação com os processos de acompanhamento.

Relativamente à prática, parece ser evidente a necessidade, nos contextos em estudo, de suprir as lacunas ao nível das informações prestadas aos acolhedores sobre as crianças a acolher, particularmente sobre a sua história familiar e os seus antecedentes de saúde bem como um maior investimento na preparação prévia ao acolhimento e no acompanhamento, tanto dos acolhedores como das crianças acolhidas.

\section{Referências}

Amorós, P., \& Palacios, J. (2004). Acogimiento familiar. Madrid, España: Alianza.

Andersson, G. (2001). The motives of foster parents, their family and work circumstances. British Journal of Social Work, 31(2), 235-248. doi:10.1093/bjsw/31.2.235

Baker, A. J. L. (2007). Client feedback in child welfare programs: Current trends and future directions. Children and Youth Services Review, 29, 1189-1200. doi:10.1016/j.childyouth.2007.05.003

Begg, I. S. (2011). Comparing fostering in Scotland and Norway with an emphasis on the use of private actors. Child Care in Practice, 17(2), 131-142. doi:10.1080/13575279.2010.5 41422

Berrick, J. D., Frasch, K., \& Fox, A. (2000). Assessing children's experiences of out-of-home care: Methodological challenges and opportunities. Social Work Research, 24(2), 119-127. doi:10.1093/swr/24.2.119

Berridge, D. (1999). Work with fostered children and their families. In M. Hill (Ed.), Effective ways of working with children and their families (pp. 240-255). London: Jessica Kingsley. doi:10.1017/s1360641700222223

Brown, J. D., \& Calder, P. (2000). Concept mapping the needs of foster parents. Child Welfare, 79(6), 729-746.

Courtney, M. E., \& Iwaniec, D. (Eds.). (2009). Residential care of children: Comparative perspectives. Oxford, UK: Oxford University Press. doi:10.1093/acprof:o so/9780195309188.001.0001

Curran, M. C., \& Pecora, P. (1999). Incorporating the perspectives of youth placed in family foster care: Selected research findings and methodological challenges. In P. A. Curtis, G. Dale Jr., \& J. C. Kendall (Eds.), The foster care crisis (pp. 99-155). Lincoln, NE: University of Nebraska Press.

Daniel, B., Wassell, S., \& Gilligan, R. (1999). Child development for child care and protection workers. London: Jessica Kingsley. doi:10.1017/s1360641799289575

Decreto-Lei $n^{\circ} 11$. (2008, 17 jan.). Diário da República, 1. ${ }^{\mathrm{a}}$ série, 12.

Del Valle, J. F., Bravo, A., \& López, M., (2009). Foster care in Spain. Its establishment and current challenges. Papeles del Psicólogo, 30(1), 33-41.

Del Valle, J. F., López, M., Montserrat, C., \& Bravo, A. (2009). Twenty years of foster care in Spain: Profiles, patterns and outcomes. Children and Youth Services Review, 31(8), 847853. doi:10.1016/j.childyouth.2009.03.007

Delgado, P. (2012). A perspectiva ecológica: Referências para a preparação e a cessação da estadia em acolhimento familiar de crianças. Psicologia: Reflexão \& Crítica, 25(2), 359-367. doi:10.1590/s0102-79722012000200018

Delgado, P. (Ed.). (2013). Acolhimento familiar de crianças. Evidências do presente, desafios para o futuro. Porto, Portugal: Livpsic.

Delgado, P., Carvalho, J. M. S., \& Pinto, V. S. (2014). Crescer em família: A permanência no acolhimento familiar. Pedagogía Social. Revista Interuniversitaria, 23, 123-150. doi:10.7179/ PSRI_2014.23.06 
Delgado, P., López, M., Carvalho, J. \& Del Valle, J. F. (2015). Acolhimento Familiar em Portugal e Espanha: Uma Investigação Comparada sobre a Satisfação dos Acolhedores.

Departamento de Desenvolvimento Social, Unidade de Infância e Juventude. (2012). Caracterização das famílias de acolhimento e das crianças e jovens em acolhimento familiar - 2011. Lisboa, Portugal: Instituto da Segurança Social.

Esping-Andersen, G. (1993). Changing classes: Social stratification in postindustrial Europe and North America. London: Sage.

Esping-Andersen, G. (2000). Fundamentos sociales de las economías postindustriales. Barcelona, España: Ariel.

Esping-Andersen, G., Garfinkel, I., Han, W. J., Magnuson, K., Wagner, S., \& Waldfogel, J. (2012). Child care and school performance in Denmark and the United States. Children and Youth Services Review, 34, 576-589. doi:10.1016/j. childyouth.2011.10.010

Fulcher, L., \& McGladdery, S. (2011). Re-examinig social work roles and tasks with foster care. Child \& Youth Services, 32(10), 19-38. doi:10.1080/0145935x.2011.553579

Gilbert, N., Parton, N., \& Skivenes, M. (Eds.). (2011). Child protection systems. New York: Oxford University Press. doi:10.1086/667366

Healy, K, Lundström, T., \& Sallnäs, M. (2011). A comparison of out-of-home care for children and young people in Australia and Sweden: Worlds apart? Australian Social Work, 64(4), 416-431. doi:10.1080/0312407x.2011.603092

Hudson, P., \& Levasseur, K. (2002). Supporting foster parents: Caring voices. Child Welfare, 81(6), 853-877.

Instituto da Segurança Social. (2013). Casa 2012. Relatório de caracterização anual da situação de acolhimento das crianças e jovens. Lisboa, Portugal: Autor.

Instituto de Desenvolvimento Social \& Comissão Nacional de Proteção de Crianças e Jovens. (2002). Crianças e jovens em acolhimento familiar. Caracterização sóciobiografica e percursos de vida. Lisboa, Portugal.

Kirton, D., Beecham, J., \& Ogilvie, K. (2004). Remuneration and performance in foster care: Report to Department for education and skills. Canterbury, UK: University of Kent.

Kirton, D., Beecham, J., \& Ogilvie, K. (2007), "Still the poor relations? Perspectives on valuing and listening to foster carers". Adoption \& Fostering, 31(3), 6-17. doi: $10.1177 / 030857590703100303$

Leibfried, S. (1993). Towards a European welfare state? In C. Jones (Ed.), New perspectives on the welfare state in Europe (pp. 133-156). London: Routledge.

López, M., Del Valle, J. F., Montserrat, C., \& Bravo, A. (2010). El acogimiento en familia ajena en España. Una evaluación de la práctica y sus resultados. Infancia y Aprendizaje, 33(2), 269-280. doi:10.1174/021037010791114616

López, M., Del Valle, J. F., Montserrat, C., \& Bravo, A. (2011). Factors affecting foster care breakdown in Spain. Spanish Journal of Psychology, 14(1), 108-118. doi:10.5209/ rev_sjop.2011.v14.n1.9

López, M., Del Valle, J. F., Montserrat, C., \& Bravo, A. (2013). Factors associated with family reunification for children in foster care. Child and Family Social Work, 18(2), 226-236. doi:10.1111/j.1365-2206.2012.00847.x

López, M., Delgado, P., Carvalho, J. M. S., \& Del Valle, J. F. (2014). Características y desarrollo del acogimiento familiar en dos países con fuerte tradición de acogimiento residencial: España y Portugal. Universitas Psichologica 13(3), 15-30. doi:10.11144/Javeriana.UPSY13-3.cdaf

Maluccio, A., Ainsworth, F., \& Thoburn, J. (2000). Child welfare outcome research in the United States, the United Kingdom, and Australia. Washington, DC: Child Welfare League of America Press.
Rhodes, K., Orme, J. G., Cox, M., \& Buehler, C. (2003). Foster family resources, psychsocial functioning and retention. Social Work Research, 27(3), 135-150. doi:10.1093/swr/27.3.135

Romaine, M., Turley, T., \& Tuckey, N. (2007). Preparing children for permanence. London: BAAF.

Samrai, A., Beinart, H., \& Harper, P. (2011). Exploring foster carer perceptions and experiences of placements and placement support. Adoption \& Fostering, 35(3), 38-49. doi:10.1177/030857591103500305

Santos, B., \& Ferreira, S. (2001). A reforma do Estado-Providência entre globalizações conflituantes. In P. Hespanha \& G. Carapinheiro (Eds.), Risco social e incerteza. Pode o Estado Social recuar mais? (pp. 177-226). Santa Maria da Feira, Portugal: Afrontamento.

Sellick, C., Thoburn, J., \& Philpot, T. (2004). What works in adoption and foster care? Ilford, UK: Barnardo's.

Sinclair, I., Gibbs, I., \& Wilson, K. (2004). Foster carers. Why they stay and why they leave. London: Jessica Kingsley. doi:10.1093/bjsw/bch172

Triseliotis, J., Borland, M., \& Hill, M. (1998). Foster carers who cease to foster. Adoption \& Fostering, 22(2), 54-61. doi: $10.1177 / 030857599802200208$

Triseliotis, J., Borland, M., \& Hill, M. (2000). Delivering foster care. London: BAAF.

Triseliotis, J., Sellick, C., \& Short, R. (1995). Foster care. Theory and practice. London: Batsford.

Verity, P. (1999). Financial matters. In A. Wheal (Ed.), Companion to foster care (pp. 192-198). Lyme Regis, UK: Russell House.

Whenan, R., Oxlad, M., \& Lushington, K. (2009). Factors associated with foster carer well-being, satisfaction and intention to continue providing out-of-home care. Children and Youth Services Review, 31(7), 752-760. doi:10.1016/j. childyouth.2009.02.001 